Nouveaux cahiers de la recherche en éducation

\title{
TraductionS - « TranslationS ». Problèmes et défis de la traduction dans le champ de l'éducation
}

Frédéric Saussez et Danièle Périsset

Volume 17, numéro 1, 2014

Traductions

Translations

URI : https://id.erudit.org/iderudit/1027318ar

DOI : https://doi.org/10.7202/1027318ar

Aller au sommaire du numéro

\section{Éditeur(s)}

Faculté d'éducation, Université de Sherbrooke

\section{ISSN}

1911-8805 (numérique)

Découvrir la revue

\section{Citer ce document}

Saussez, F. \& Périsset, D. (2014). TraductionS - « TranslationS ». Problèmes et défis de la traduction dans le champ de l'éducation. Nouveaux cahiers de la recherche en éducation, 17(1), 1-11. https://doi.org/10.7202/1027318ar d'utilisation que vous pouvez consulter en ligne. 


\section{Éditorial}

\section{TraductionS - «TranslationS" \\ Problèmes et défis de la traduction dans le champ de l'éducation}

\section{Frédéric Saussez}

Université de Sherbrooke

\section{Danièle Périsset}

Université de Genève

Numéro thématique conjointement construit et publié par la revue en ligne Nouveaux c@hiers de la recherche en éducation (www.usherbrooke.ca/ncre/) et la Revue suisse des sciences de l'éducation ${ }^{1}$ (www.rsse.ch).

\section{En guise de préambule: pourquoi un numéro conjoint entre deux revues scientifiques?}

La revue Nouveaux c@hiers de la recherche en éducation (NCRÉ) célèbre cette année son vingtième anniversaire. Pour marquer cet événement, les NCRÉ ont mené différents projets internationaux visant la mise en réseau de revues scientifiques généralistes en éducation. Outre la réalisation de ce numéro conjoint avec la Revue suisse des sciences de l'éducation (RSSE), les NCRÉ ont également amorcé un partenariat avec la revue Estudios Pedagógicos publiée par la Facultad de Filosofia y Humanidades de l'Universidad Austral du Chili. Celui-ci a pour objectif, à partir de l'année 2015-2016, de traduire en espagnol, une fois par an, un article représentatif de l'évolution actuelle de la recherche québécoise en éducation publié dans la revue NCRÉ afin de le diffuser dans la revue Estudios Pedagógicos et, inversement, de traduire en français, une fois par an, un article représentatif de l'évolution actuelle de

$1 \quad$ Exceptionnellement et en l'honneur de cette collaboration, ce numéro thématique sera immédiatement mis en ligne sur le site de la RSSE. II sera donc téléchargeable depuis les sites respectifs des deux revues scientifiques, RSSE et NCRÉ. À noter que bien que la revue Nouveaux c@hiers de la recherche en éducation soit une revue scientifique francophone, les articles du présent numéro thématique y seront publiés dans leur langue originale. 
la recherche chilienne publié dans la revue Estudios Pedagógicos afin de le diffuser dans la revue NCRÉ.

De son côté, la Revue suisse des sciences de l'éducation est forte de plus de 20 ans de publication, au sein d'une même revue, de travaux issus des trois courants culturels (francophone, germanophone, italophone) dont la Suisse est faite. L'occasion était belle d'élargir encore ce processus en s'engageant dans cette collaboration internationale (et intercontinentale) sur un objet qui est au cœur même de la nature de son travail éditorial interculturel, à savoir les enjeux de traduction (sur lesquels nous revenons plus loin).

La mise en place d'une collaboration rédactionnelle entre ces revues participe aussi d'une volonté d'accroître le rayonnement international de chacune. Elle vise en outre à favoriser une plus grande compréhension des chercheurs à propos des enjeux de la recherche en éducation dans leurs contextes respectifs et à stimuler la mobilité des savoirs produits dans ceux-ci.

Ces collaborations rédactionnelles s'inscrivent également dans une perspective plus politique. En effet, dans le champ de l'éducation, tout comme dans les autres champs des sciences humaines et sociales, les revues se sont imposées comme un moyen privilégié de diffuser les résultats de recherche. Néanmoins, depuis une trentaine d'années, le domaine de l'édition scientifique connait de profondes transformations et est dominé par une logique marchande se caractérisant notamment par sa concentration aux mains de quelques éditeurs. Une telle concentration fait courir le risque d'une homogénéisation des thématiques, des cadres de référence et des méthodes de recherche dans le champ de la recherche en éducation. En outre, elle encourage principalement les publications en langue anglaise. Or, la recherche en éducation présente une forte composante politique, culturelle et linguistique. Face à de tels développements, il est de plus en plus difficile pour des éditeurs institutionnels de se développer en proposant tout à la fois un contenu dans une autre langue que l'anglais, une diversification du lectorat et un ancrage fort dans la réalité socioépistémologique, culturelle et linguistique de leur propre société. L'enjeu de ces projets et notamment de la réalisation de ce numéro conjoint est de mettre en place des conditions propices à la mise en visibilité de problèmes et de manières de faire de la recherche en éducation propres à différents contextes nationaux et internationaux, européens ou nordaméricains, ceci dans les langues nationales que sont le français, l'allemand et l'anglais, le 
français étant langue nationale au Canada comme en Suisse, les deux autres langues étant spécifiques (et dominantes) à l'un ou l'autre pays.

\section{Problèmes et défis de la traduction dans le champ de l'éducation}

Lors d'un premier contact entre les deux comités de rédaction, le thème de la traduction s'est naturellement imposé comme une évidence pour structurer le projet d'un numéro thématique conjoint. Ne sommes-nous pas confrontés dans nos pays respectifs comme chercheurs et comme citoyens à une diversité de langues et de cultures? Celle-ci ne nous place-t-elle pas face à des problèmes et des défis particuliers? Néanmoins, au fil des échanges entre les deux personnes mandatées par leur comité de rédaction respectif pour concrétiser ce projet, la question de la traduction s'est également posée dans ses rapports avec la mise en circulation des résultats de la recherche dans une économie du savoir et les transformations majeures que celle-ci introduit dans les modes de gouvernance des états et des systèmes éducatifs.

Dans un contexte de globalisation et de mise en place du capitalisme cognitif (MoulierBoutang, 2007), la recherche et le développement scientifique occupent une place centrale. Ils sont présentés comme des secteurs clés de l'économie du savoir. Le champ de l'éducation n'est pas en reste. II est concerné par cette évolution, autant au plan de la conception, de la mise en œuvre et de l'évaluation des dispositifs de formation des acteurs individuels et collectifs aux compétences et exigences requises par cette économie, qu'au plan de la production, de la mise en circulation et de la mise en marché des savoirs qui y sont produits. Quels sont les problèmes, les défis et les enjeux posés à la recherche en éducation par les opérations de traduction engendrées par ce nouvel ordre économique mondial?

Cette question constitue un des axes de ce numéro thématique consacré aux problèmes et défis posés par la traduction dans le champ de l'éducation. Ceux-ci ne concernent donc pas strictement la circulation des œuvres scientifiques au sein d'une zone géographique, temporelle, linguistique, culturelle ou sociale marquée du sceau de la diversité des langues et des cultures. La traduction dans le contexte de l'économie des savoirs implique aussi de se pencher sur la reconfiguration des rapports entre production et utilisation des savoirs ainsi que sur les différentes opérations de traduction à l'œuvre dans le processus de mise en 
interface entre producteurs, utilisateurs et commanditaires de savoirs. Enfin, les problèmes et les défis de la traduction concernent également la façon dont se concrétisent les principes d'une économie néolibérale dans une politique éducative et de multiples projets de transformation de l'École et de l'Université pour les mettre au service de ce nouvel ordre économique mondial. Ainsi, dans ce numéro conjoint entre la revue NCRÉ et la RSSÉ, les problèmes et défis de la traduction dans le champ de l'éducation sont traités à travers trois niveaux d'analyse.

\section{Traduire, un travail de mise en rapport interculturel?}

La question de la traduction est une question sensible dans les pays où la diversité des langues est une réalité comme dans les deux pays concernés par ce numéro conjoint à savoir le Canada et la Confédération helvétique. Sans nier le fait que les débats autour des langues nationales constituent souvent des analyseurs des rapports sociaux entre groupes linguistiques, nous avons initialement envisagé la question de la traduction en éducation dans une perspective culturaliste. Les problèmes et défis soulevés par celle-ci concernent alors davantage, à un premier niveau d'analyse, la mise à disposition d'une œuvre produite dans une des langues nationales aux membres d'une autre communauté linguistique. Le texte à traduire pose une série de problèmes et de défis qui excède largement les simples opérations de décodage et de codage. Il s'agit certes de trouver les mots pour le traduire. Cependant, il s'agit aussi tout autant de faire pénétrer un lecteur dans un contexte culturel de production étranger que de faire voyager une œuvre dans un nouveau contexte interprétatif. Sous cet angle, traduire consiste sans doute à médiatiser un rapport interculturel. En effet, le texte possède une épaisseur sociale, historique, culturelle, politique qui contribue à lui conférer un sens particulier et il doit aussi se situer dans un nouveau cadre interprétatif. Somme toute, le travail de traduction ouvre un espace-temps à une forme d'exotopie au sens entendu par Bakhtine (1984):

Dans le domaine de la culture, l'exotopie est le moteur le plus puissant de la compréhension. Une culture étrangère ne se révèle dans sa complétude et dans sa profondeur qu'au regard d'une autre culture (et elle ne se livre pas dans toute sa plénitude, car d'autres cultures viendront qui verront et comprendront davantage encore). Un sens se révèle dans sa profondeur pour avoir rencontré et s'être frotté à un autre sens, à un sens étranger: entre les deux s'instaure comme un 
dialogue qui a raison du caractère clos et univoque, inhérent au sens et à la culture pris isolément. À une culture étrangère, nous posons des questions nouvelles telles qu'elle-même ne se les posait pas. Nous cherchons en elle une réponse à ces questions qui sont les nôtres, et la culture étrangère nous répond, nous dévoilant ses aspects nouveaux, ses profondeurs nouvelles de sens. Si nous ne posons pas nos propres questions, nous nous coupons d'une compréhension active de tout ce qui est autre et étranger (il s'agit, bien entendu, de questions sérieuses, vraies). La rencontre dialogique de deux cultures n'entraîne pas leur fusion, leur confusion - chacune d'elle garde sa propre unité et sa totalité ouverte, mais elles s'enrichissent mutuellement. (p. 348-349)

Cet axe s'articule autour d'une conception du langage qui s'inscrit en porte à faux de la conception d'un langage tuyau qui servirait simplement à transporter des informations d'un émetteur à un récepteur et à remplir une simple fonction de communication (Wertsch et Penuel, 1998), il s'agit d'un instrument de pensée, de construction d'un rapport au monde et d'un rapport à la pensée d'autrui comme invite à le penser les contributions de Samain et de Schwimmer. Mais ces deux auteurs invitent à pousser plus loin la réflexion sur la nature du langage et à conceptualiser le travail de traduction en termes de construction d'un sens nouveau pour le premier ou de créativité et d'engagement pour le second.

Samain souligne le fait qu'un texte s'inscrit toujours dans un rapport au monde particulier. Il signifie donc toujours plus que ce qu'il renferme. Par conséquent, le travail de traduction, de reformulation ne peut faire l'impasse sur ce rapport de sens entre texte et contexte. Ainsi, traduire consisterait à aménager un passage propice à la migration de notions d'une structure de signification à une autre, c'est contribuer aussi au développement d'un métalangage propre. Dans une perspective dialogique, nous serions tentés d'écrire que traduire c'est ouvrir un nouvel espace-temps de pensée et reconfigurer le sens en l'inscrivant dans un nouvel horizon interprétatif. Pour Samain, dans le travail de traduction, «analyser un énoncé, c'est lui faire subir un changement d'état logique». Ce processus s'inscrit bien dans un rapport entre entropie et néguentropie. Il y a bien perte d'une signification possible, mais aussi surplus de sens.

La contribution de Samain invite aussi à penser la question de la traduction autrement qu'en référence au passage d'un texte d'un univers linguistique à un autre. Les opérations de traduction/reformulation concernent également les passages de notions/concepts d'un 
univers conceptuel à un autre. La traduction, en opérant à la fois sur le texte et sur le rapport au monde qu'il institue, greffe des notions dans de nouvelles structures de sens. Il s'agit d'une perspective stimulante pour penser la position particulière des sciences de l'éducation au regard des autres disciplines en sciences humaines et sociales auxquelles elles puisent abondamment des ressources conceptuelles tout en devant les détacher de leur structure de sens pour les faire signifier dans un rapport à l'éducation comme objet de recherche et non comme terrain d'application de concepts forgés pour faire parler d'autres sphères du monde social.

Le long de cet axe, la contribution de Dugonjić et Richard de Latour éclaire le contexte politique dans lequel opère le travail de traduction et le marché de la traduction. Ces auteures rappellent combien les langues - nous serions tentés d'écrire et aussi les différents sociolectes au sein d'une même société - s'inscrivent dans des rapports de valeur et de domination et que sous couvert d'une ouverture à d'autres cultures, la traduction des œuvres de la littérature mondiale fait aussi fonctionner une certaine forme d'impérialisme culturel: on traduit d'une langue dominante vers d'autres langues, mais pas l'inverse. En décalage et de façon très naïve, nous sommes tentés de formuler sous la forme d'une question une idée paradoxale en croisant ces deux textes: la position dominante d'une langue ne constitue-t-elle pas à terme, un facteur d'appauvrissement, d'entropie en ralentissant les flux migratoires de concepts étrangers en mesure de venir retravailler de l'intérieur les cadres interprétatifs en vigueur au sein de cette position?

\section{Traduire la recherche pour les praticiens entre normalisation et créativité}

À un deuxième niveau d'analyse, il nous semble important de poser la question de la traduction en la situant au regard de l'entreprise scientifique de production du savoir dans ses rapports complexes avec les commanditaires et les utilisateurs. La notion de mode 2 de production de connaissances (Gibbons et al., 1994), bien que contestée, force à réfléchir à la reconfiguration de ces rapports et aux opérations de traduction à l'œuvre au sein de différentes interfaces entre recherche, politique et profession. Ainsi, la qualité du travail de production de savoir est évaluée en référence à des exigences non seulement de validité scientifique, mais aussi de pertinence sociale. Le champ des sciences de l'éducation est 
soumis à des dynamiques contradictoires liées, d'une part, à la quête d'autonomie inhérente à l'entreprise scientifique et, d'autre part, à la volonté des commanditaires et des utilisateurs de peser sur la façon dont les problèmes pratiques sont pris en compte par la recherche (Saussez, Dulude, Dembélé, Lessard, Maroy et Yvon, 2014).

Sous cet angle, la traduction concerne deux processus: la traduction pragmatique (Hammersley, 2002) des résultats de recherche en guides d'action destinés aux professionnels de l'éducation et la traduction épistémologique, la réfraction (Bourdieu, 2001) en une sémantique d'intelligibilité des problèmes issus des pratiques éducatives et formulés initialement à l'aide des catégories d'une sémantique de l'action (Barbier, 2000) pour construire un questionnement scientifique spécifique au champ de l'éducation. Dans quelle mesure le débat récurrent qui traverse le champ de la recherche en éducation concernant tout à la fois sa scientificité et sa capacité à contribuer à l'amélioration des pratiques et des politiques éducatives ne constitue-t-il pas un analyseur de la difficulté de ce champ à se structurer de façon autonome autant au regard de disciplines contributives qu'au regard des demandes et attentes issues des milieux professionnels et politiques (Saussez et al., 2014)?

Ce débat a été ravivé dans la dernière décennie avec le développement et I'institutionnalisation de l'approche de l'éducation basée sur des données probantes (Saussez et Lessard, 2009). Cette approche fournit "une orientation précise concernant la façon dont la recherche est en mesure de contribuer à l'amélioration des pratiques et des politiques éducatives. Elle procède de la conviction que l'éducation peut et doit être soumise à la rationalité instrumentale, qu'il importe d'accroître la précision, l'efficacité et l'efficience du noyau technique des différents intervenants en leur fournissant des guides d'action fondés sur des données probantes (Saussez et al., accepté)". Elle incarne une conception particulière des rapports entre recherche et pratique. Les orientations propres à cette approche ne conduisent-elles pas à une vision de la traduction obéissant à la rationalité instrumentale, sans qu'apparaisse nécessaire et légitime un travail interprétatif des acteurs individuels et collectifs concernés, comme Hammersley invite à le penser? Comment, sans nécessairement verser dans une conception postmoderniste de la science, faire valoir la complexité de l'action professionnelle et problématiser le pouvoir créatif du praticien dans le travail de traduction de données probantes en des perspectives d'action comme Schwimmer le suggère? 
Hammersley propose de revisiter la métaphore de la traduction telle qu'elle s'est développée sous l'influence du fonctionnalisme. Celle-ci repose sur un modèle des rapports entre la recherche et la politique ou la pratique de type ingénierie et sur une vision de la traduction comme une opération transparente, non problématique permettant un transfert optimal des résultats de recherche vers des acteurs rationnels. Dans ce contexte, le fossé entre la recherche et la pratique peut être comblé. Bien qu'il ne la nomme pas, Hammersley revisite cette métaphore en référence à la théorie des deux mondes: chercheurs et praticiens vivent dans deux mondes distincts, ont des préoccupations distinctes et mobilisent des langages distincts (Hammersley, 2002). Il est donc illusoire de penser que ces deux mondes pourraient fusionner. Pour Hammersley, il faut prendre au sérieux l'existence de ces deux mondes et sur cette base revisiter la métaphore de la traduction et lui donner un sens proche de l'interprétation. Il importe de concevoir le praticien comme exerçant un jugement, s'engageant dans des délibérations individuelles et collectives concernant le choix de moyens les plus appropriés pour faire face à une situation aux regards de diverses considérations. Dans cette perspective, si la recherche a bien pour fonction de produire de nouveaux schémas de pensée, elle ne peut contribuer au développement des pratiques que de façon indirecte.

Schwimmer s'intéresse également au problème des rapports entre recherche et pratique. Elle ne réfute pas l'intérêt d'une vision culturaliste de la traduction et d'une lecture en référence à la théorie des deux mondes. Toutefois, elle tente de recadrer la question à l'aide d'une conception derridienne du langage reposant sur la thèse d'un travail permanent de traduction lié à la nature du langage, à son impropriété. Pour Schwimmer, la traduction correspond alors à "une transformation et un renouvellement d'une chose vivante". Somme toute, la réalité sémiotique du langage nous condamne à un travail permanent de traduction. Dans le cadre de l'analyse de la traduction de la recherche vers la pratique, cette conception met au centre de ce travail le praticien et souligne le caractère imprévisible de cette traduction. Celle-ci ne résulte pas comme dans le texte d'Hammersley, de l'appartenance des personnes à des mondes culturels distincts, mais de la nature même du langage. Ces deux auteurs se rejoignent pourtant sur une des conséquences de leur conceptualisation respective de la traduction. Elle implique chacune un investissement dans le jugement des acteurs. Schwimmer propose alors de prendre en compte cette donnée pour évaluer la 
qualité d'une recherche scientifique et place le chercheur devant le défi de produire des recherches inspirantes!

\section{Prolifération des projets de transformation des systèmes éducatifs: traduction des principes d'une économie néolibérale dans le champ de la politique éducative?}

À un troisième niveau d'analyse, il s'agit de traiter de manière explicite de la traduction des principes d'une économie néolibérale et du capitalisme cognitif dans une politique éducative relayée par différents organismes supranationaux (OCDE, Banque mondiale, Fonds monétaire international, etc.). En dépit des formes et des contenus particuliers des réformes éducatives mises en place depuis une quinzaine d'années dans les pays de l'OCDE, ne sommes-nous pas en présence d'une politique éducative traduisant les principes du néolibéralisme? Comment de tels impératifs pénètrent-ils les systèmes éducatifs nationaux?

La contribution de Kiuppis illustre bien la façon dont des organismes supranationaux tentent de façonner les politiques éducatives des états. Cet auteur propose une conceptualisation, à partir d'une analyse du contenu des archives de l'OCDE, des processus de traduction d'une idée tout au long du développement d'un projet ayant pour objet l'accès à l'éducation de public à risques. Ce projet visait à fournir aux états membres des orientations pour leurs politiques nationales. Un des apports de ce texte est la mise en lumière de différentes opérations à travers lesquelles des savoirs scientifiques sont en mesure de prendre sens ou non au regard du contexte social, culturel et politique dans lequel ils s'insèrent. Ainsi Kiuppis est en mesure de tracer certaines des transformations affectant des idées clés d'un projet en référence à la façon dont celles-ci étaient jugées compatibles ou non par les acteurs politiques aux regards des particularismes nationaux.

Nóvoa, Carvalho et Yanes tentent de cerner une transformation en cours des modes d'influence d'organismes supranationaux sur les politiques éducatives des états à travers la façon dont ils traduisent les résultats de comparaisons internationales en orientations politiques visant la transformation des systèmes éducatifs afin de les ajuster aux exigences d'une société de la connaissance. Ces auteurs esquissent de cette façon, les contours d'un domaine de recherche en friche. Ils discutent notamment l'idée que le capital intellectuel 
et la qualité des processus de sa fabrication sont traduits en indicateurs de performances des différents systèmes éducatifs. Ces indicateurs sont agrégés afin de produire des indices concernant la santé et la vitalité de la nouvelle économie des différents états, influencent leur notation par les grandes agences de notation et, in fine, sont en mesure de conditionner les possibilités d'obtention de financement et de crédit. Ainsi, selon ces auteurs, la traduction des résultats des comparaisons internationales amorce un tournant majeur dans la façon dont elle contribue à la reconfiguration des politiques publiques au plan national, à l'adoption des instruments de la gestion axée sur les résultats. Nóvoa et al. appellent à une analyse critique du travail opéré dans ces espaces transfrontaliers où se fabriquent et prolifèrent de nouveaux instruments d'action publique et où se jouent les nouvelles formes du capitalisme cognitif.

Au terme de la lecture de ce numéro thématique, d'aucuns pourraient nous reprocher de ne pas avoir proposé de contributions inspirées de la sociologie de la traduction, de ne pas avoir sollicité de lecture de la didactique en termes de traduction ou encore de ne pas avoir discuté de tout un courant de recherche aux États-Unis d'Amérique qui s'intéresse à l'usage des données probantes par différentes catégories d'acteurs du monde de l'éducation (voir Coburn et Stein, 2010). Nous sommes bien conscients que de multiples approches de la traduction se développent dans différents champs des sciences humaines et sociales. Ce numéro thématique est un premier défrichement. Il appelle d'autres contributions pour cerner les Traductions dont est l'objet le paradigme de la traduction lorsque ses concepts pénètrent les systèmes de sens propres aux sciences de l'éducation.

\section{Post-scriptum}

La collaboration rédactionnelle entre les deux revues a conduit aussi à réfléchir sur la façon dont la citation des contributions pourrait lui rendre justice et, ce faisant, offrir également un peu de résistance aux modalités usuelles de référencement. Nous invitons les personnes utilisant les différentes contributions composant ce numéro à les citer de la façon suivante: 
Saussez, F. et Périsset, D. (2014). Traductions - "TranslationS». Problèmes et défis posés par la traduction dans le champ de l'éducation. Les Nouveaux c@hiers de la recherche en éducation, 17(1), 2014) (numéro conjoint avec la Revue suisse des sciences de l'éducation, 36(2), 2014).

\section{Références}

Bakhtine, M. (1984). Esthétique de la création verbale. (Trad. par A. Aucouturier). Paris: NRF. (Ouvrage original publie en 1978).

Barbier, J-M. (2000). Sémantique de l'action et sémantique d'intelligibilité des actions. Le cas de la formation. In B. Maggi (Éd.), Manière de penser, manière d'agir en éducation et en formation (p. 89-104). Paris: PUF.

Bourdieu, P. (2001). Science de la science et réflexivité. Paris : Raisons d'agir.

Coburn, C E. et Stein, M. K., (Éd.). (2010). Research and practice in education: Building alliances, bridging the divide. NY: Rowman \& Littlefield Publishing Group.

Gibbons, M., Limoges, C., Nowotny, H., Schwartzman, S., Scott, P. et Trow, M. (1994). The new production of knowledge. The dynamics of science and research in contemporary societies. London: Sage.

Hammersley, M. (2002). Educationnal research and evidence based practice. London: Sage.

Moulier-Boutang, Y. (2007). Le capitalisme cognitif: la nouvelle grande transformation. Paris: Éditions Amsterdam.

Saussez, F. et Lessard, C. (2009). Entre orthodoxie et pluralisme, les enjeux de l'éducation basée sur la preuve. Revue française de pédagogie, 168, 111-137.

Saussez, F., Dulude, E., Dembélé, M., Lessard, C., Maroy, C. et Yvon, F. (2014). Regard critique sur la reconfiguration des rapports entre recherche, politique et pratique à l'œuvre dans l'éducation basée sur la preuve. Texte mis en circulation dans le cadre du colloque 553 - La recherche et le politique en éducation: enjeux et débats dans le monde francophone organisé dans le cadre du congrès de l'ACFAS qui s'est tenu à Montréal les 12 et 13 mai 2014.

Saussez, F., Dulude, E., Dembélé, M., Lessard, C., Yvon, F. et Maroy, C. (accepté). Lapromotion de l'éducation basée sur des données probantes au Canada: le cas du Conseil Canadien sur I'Apprentissage. À paraître dans la revue Éducation comparée.

Wertsch, J. et Penuel, W. (1998). The individual-society antinomy revisited: productive tensions in theories of human development, communication and education. In $\mathrm{D}$. Olson et N. Torrance (Ed.), The handbook of education and human development (p. 415-433). Oxford: Blackwell. 